\title{
Health-Related Quality of Life in Children and Adolescents with Severe Obesity after Intensive Lifestyle Treatment and at 1-Year Follow-Up
}

\author{
Meeke Hoedjes ${ }^{a}$ b Sabine Makkes ${ }^{a}$ Jutka Halberstadt ${ }^{a}$ \\ Hanneke Noordam ${ }^{a}$ Carry M. Renders ${ }^{a}$ Judith E. Bosmans ${ }^{a}$ \\ Olga H. van der Baan-Slootweg ${ }^{c}$ Jacob C. Seidella \\ ${ }^{a}$ Amsterdam Public Health Research Institute, Department of Health Sciences, Vrije \\ Universiteit Amsterdam, Amsterdam, the Netherlands; ${ }^{b}$ Center of Research on Psychology \\ in Somatic Diseases, Department of Medical and Clinical Psychology, Tilburg University, \\ Tilburg, the Netherlands; ${ }^{\mathrm{C}}$ Merem Childhood Obesity Center, Heideheuvel, Hilversum, the \\ Netherlands
}

\section{Keywords}

Health-related quality of life · Severe childhood obesity · Lifestyle treatment

\begin{abstract}
Objective: To examine changes in generic and weight-related, health-related quality of life (HRQoL) in children and adolescents with severe obesity participating in intensive lifestyle treatment, and to examine whether changes in SDS-BMI were associated with changes in HRQoL. Methods: In this prospective observational study, a referred sample of 120 children and adolescents (8-19 years) with severe obesity (SDS-BMI $\geq 3.0$, or $\geq 2.3$ in combination with obesity-related comorbidity) received an intensive 1-year lifestyle treatment with an inpatient period in a specialized childhood obesity center. A weight-related (IWQOL-Kids) and three generic (KIDSCREEN-52, PedsQL 4.0, and EuroQol) HRQoL questionnaires were administered at baseline (T0), after treatment (T1), and 1 year later (T2). Generalized Linear Mixed Models and partial correlations were used to analyze changes in HRQoL and associations with changes in SDS-BMI. Results: Statistically significant improvements in generic and weight-related HRQoL overall and domain scores were observed at T1and at T2 in comparison with T0, despite partial weight regain from T1 to T2. Larger weight loss at T2 was correlated with larger improvements in physical HRQoL domains. Conclusion: Children and adolescents with severe obesity experienced long-term improvements in generic and weight-related HRQoL after participating in intensive lifestyle treatment, despite partial weight regain.
\end{abstract}


Hoedjes et al.: Health-Related Quality of Life in Children and Adolescents with Severe Obesity after Intensive Lifestyle Treatment and at 1-Year Follow-Up

\section{Introduction}

Childhood obesity is associated with somatic health problems and with negative selfevaluation, bullying, social stigma, symptoms of depression and anxiety, resulting in a negative impact on physical and psychosocial health in children and adolescents [1-6]. Compared with children and adolescents with overweight and obesity, children and adolescents with severe obesity have a higher cardiometabolic risk [7]. Moreover, children and adolescents with severe obesity frequently report an impaired health-related quality of life (HRQoL) [8], which can be defined as 'the impact of health or disease on physical, mental, and social well-being from the patient's point of view' [9]. Previous studies in children and adolescents with severe obesity have indicated that intensive lifestyle treatment may result in improvements in overall HRQoL and in the physical, mental and social wellbeing domains [10-13].

HRQoL can be assessed with both generic and condition-specific measures. Whereas generic measures contain general HRQoL items that are applicable to a wide variety of populations, condition-specific measures contain items on the characteristics and complaints that are particularly relevant to a specific condition, such as obesity. Weight-related HRQoL can be measured to assess condition-specific HRQoL in individuals with obesity. Previous research suggests that weight-related HRQoL is more sensitive to the physical and psychological impact of obesity and may be more responsive to weight loss after intensive lifestyle treatment than generic HRQoL $[10,14]$. Since generic and condition-specific measures assess different aspects of HRQoL, it has been recommended to evaluate both distinct, complementary concepts in studies among children and adolescents with obesity $[10,15,16]$.

Since the number of severely obese children and adolescents is increasing [7] and common treatment options are less effective in those with severe obesity $[17,18]$, there is a need for novel treatment options for this specific target population. However, there is a lack of studies examining the long-term effects of intensive lifestyle treatments on HRQoL $[10,13]$ in children and adolescents with severe obesity [7]. Moreover, little is known about the effects of intensive lifestyle treatment on the different domains of HRQoL [12]. Such knowledge can provide valuable information on the effectiveness of these treatments in children and adolescents with severe obesity [19].

The main objective of this study was to examine changes in generic and weight-related HRQoL directly after treatment and at follow-up 1 year later in children and adolescents with severe obesity participating in an intensive 1-year lifestyle treatment including an inpatient period. We hypothesized an increase in HRQoL after intensive lifestyle treatment, particularly in weight-related HRQoL. A secondary objective was to examine whether changes in Standard Deviation Score of Body Mass Index (SDS-BMI) were associated with changes in generic and weight-related HRQoL. We expected a positive association between changes in SDS-BMI and changes in HRQoL, particularly changes in weight-related HRQoL.

\section{Material and Methods}

Study Design and Population

This study (HELIOS, Netherlands Trial Register 1678) was a prospective observational study of 120 children and adolescents (8-19 years) with severe obesity receiving an intensive 1-year lifestyle treatment with an inpatient period (of either 2 or 6 months) at a specialized childhood obesity center in Hilversum, the Netherlands (Childhood Obesity Centre Heideheuvel) [20,21]. Severe obesity was defined as a SDS-BMI $\geq$ 3.0 (99.9th age- and sex-specific percentile of BMI in the fourth Dutch nationwide growth study of 1997) or a SDS-BMI $\geq 2.3$ (99th age- and sex-specific percentile of BMI in the fourth Dutch nationwide growth study of 1997) in combination with obesity-related comorbidity [20]. Measurements were conducted at baseline 
Hoedjes et al.: Health-Related Quality of Life in Children and Adolescents with Severe Obesity after Intensive Lifestyle Treatment and at 1-Year Follow-Up

(T0), directly after treatment (at 12 months after baseline, T1), and at follow-up (24 months after baseline, 12 months after treatment, T2).

Details on study design, study population, and lifestyle treatment can be found elsewhere [20, 21]. Written informed consent was obtained from both the participants and their parents. The informed consent procedure, the study design, and the study protocol were approved by the Medical Ethics Committee of VU University Medical Center Amsterdam.

\section{Measurements}

HRQoL Questionnaires

Dutch versions of child self-report questionnaires with adequate psychometric properties were used to assess HRQoL [22-28]. To assess generic HRQoL, the KIDSCREEN-52 [22, 23], Pediatric Quality of Life Inventory (PedsQL 4.0) [25], and EuroQol (EQ-5D-3L and EQ-VAS) [25] were used. Each instrument measures generic HRQoL in a different manner, and detects different relevant generic HRQoL domains. Since it is currently unknown which of these measures is most suitable for the measurement of changes in generic HRQoL in children and adolescents with severe obesity participating in intensive lifestyle treatment, we used multiple questionnaires to assess generic HRQoL to be able to provide a recommendation on the suitability of those questionnaires.

The KIDSCREEN-52 [21, 22] (8-18 years) contains 52 items divided among 10 domains: 1) physical well-being (e.g., Thinking about the last week, have you felt fit and well?); 2) psychological well-being; 3) moods and emotions; 4) self-perception; 5) autonomy; 6) relations with parents and home life; 7) social support and peers; 8) school environment; 9) social acceptance (bullying); and 10) financial resources. Each item is rated using a 5-point Likert-type scale assessing either the frequency (never, seldom, quite often, very often, always) or the intensity (not at all, slightly, moderately, very, extremely) of certain behaviors, feelings, or attitudes, based on a recall period of 1 week. Summary scores were calculated and Rasch Person Parameters (PP) were assigned to each possible summary score [22]. The PPs were transformed into T-values with a mean of 50 and a standard deviation (SD) of approximately 10. Higher scores indicate better HRQoL. Since the KIDSCREEN-52 does not provide a one-dimensional overall measure of generic HRQoL, KIDSCREEN-10 index scores were additionally derived from the KIDSCREEN-52 [23]. Similar to scoring of the KIDSCREEN-52, KIDSCREEN-10 scores were also transformed into T-values. Please see Ravens-Sieberer et al. [23] for more information on how the KIDSCREEN-10 score is obtained from the KISCREEN-52.

The PedsQL 4.0 [24] contains 23 items and consists of four domains: 1) physical functioning (e.g., It is hard for me to run); 2) emotional functioning (e.g., I feel sad); 3) social functioning (e.g., I have trouble getting along with peers); 4) school functioning (e.g., I miss school because I do not feel well). The PedsQL 4.0 assesses how much of a problem each item has been during the past month with a 5-point Likert-type scale varying from 0 (never a problem) to 4 (almost always a problem). Scores are transformed on a scale from 0 to 100 . Three summary scores can be calculated, with higher scores indicating a better HRQoL: a Total Scale Score (23 items), a Physical Health Summary Score (i.e., 8 items from the physical functioning domain), and a Psychosocial Health Summary Score (15 items: items from the emotional, social, and school functioning domains).

The EuroQol consists of the EQ-5D-3L descriptive system (EQ-5D-3L) and the EQ visual analogue scale (EQ-VAS). The EQ-5D-3L measures generic HRQoL on the day of completion in five dimensions (mobility, self-care, usual activities, pain/discomfort, and anxiety/depression) with three levels of severity (no problems / some or moderate problems / extreme problems) [26]. The resulting health status was transformed to a utility score using the Dutch EQ-5D valuation tariff [27]. Utilities represent generic HRQoL in a single number, ranging from 0.0 (death) to 1.0 (full health). The EQ-VAS consists of a vertical $20 \mathrm{~cm}$ visual analogue scale to measure an individual's direct valuation of their current generic HRQoL on a scale of 0 (worst imaginable health status) to 100 (best imaginable health status) [26].

To assess weight-related HRQoL, the Impact of Weight on Quality of Life-Kids (IWQOL-Kids) was used. The IWQOL-Kids [15, 28] contains 27 items on four domains: 1) physical comfort; 2) body esteem; 3) social life; and 4) family relations. Each item begins with the phrase 'Because of my weight' (e.g., 'Because of my weight, I avoid looking at myself in mirrors or on pictures') and contains five response options, ranging from 'always' (1) to 'never' (5). The recall period is 1 week. IWQOL-Kids total and subscale scores range from 0 to 100 , with higher scores indicating a better HRQoL. 
Hoedjes et al.: Health-Related Quality of Life in Children and Adolescents with Severe Obesity after Intensive Lifestyle Treatment and at 1-Year Follow-Up

Table 1. Baseline characteristics of the study population

\begin{tabular}{ll}
\hline & $\begin{array}{l}\text { Total } \\
(\mathrm{N}=120)\end{array}$ \\
& $14.8(2.4)$ \\
Mean age, years (SD) & $81(67.5)$ \\
Female, $\mathrm{n}(\%)$ & $76(63.3)$ \\
Ethnicities, \% of total & $41(34.2)$ \\
$\quad$ Western & \\
$\quad$ Non-Western & $44(36.7)$ \\
Educational level of the parents/caregivers, n (\% of total) & $47(39.2)$ \\
$\quad$ Low & $23(19.2)$ \\
$\quad$ Medium/intermediate & $73(60.8)$ \\
$\quad$ High & $41(34.2)$ \\
SES, n (\% of total) & $62(51.7)$ \\
$\quad$ Below average & $38(31.7)$ \\
Above average & $14(11.7)$ \\
Household situation, $\mathrm{n}(\%$ of total) & $6(5.0)$ \\
$\quad$ Married/living together & \\
$\quad$ Divorced & $80(66.7)$ \\
One parent family (mother) & $40(33.3)$ \\
Other situation & $3.4(0.4)$ \\
Duration of inpatient treatment program, $\mathrm{n}(\%)$ &
\end{tabular}

$\mathrm{SD}=$ Standard deviation; SDS-BMI = standard deviation score of body mass index; $\mathrm{SES}=$ socioeconomic status.

Demographic and Participant Characteristics

Demographic characteristics were assessed at baseline. Ethnicity of participants was categorized as: Western, including native Dutch as well as Western immigrants; and Non-Western, including all Non-Western immigrants. If both parents were born abroad but in different countries, the country in which the mother was born was used to categorize the participant [31].

Highest educational level attained by one of the parents/caregivers was categorized into low, medium/ intermediate, or high [32, 33].

To determine socioeconomic status (SES), data from The Netherlands Institute for Social Research was used to obtain status scores of the parents [34]. A status score is a measure for the social status of a postal code area and consists of three components: income, level of education, and level of unemployment. A status score below 0 represents a SES above average and a status score above 0 represents a SES below average $(0$ represents average) [34]. SES was dichotomized into 'above average' and 'below average'.

Household situation was dichotomized into 'parents living together' ('married / living together') and 'parents not living together' ('divorced', 'one-parent family', and 'other situation').

BMI was calculated as weight divided by square height $\left(\mathrm{kg} / \mathrm{m}^{2}\right)$. SDS-BMIs were calculated with the Growth Analyser [35] using the fourth Dutch nationwide growth study of 1997 as reference.

Statistical Analyses

Statistical analyses were performed using IBM SPSS Statistics for Windows, Version 20 (IBM, Armonk, NY, USA).

Mean and frequency tables were used to describe baseline characteristics. Differences between completers of treatment and dropouts of treatment were tested using independent Student's t-tests for continuous variables and chi-square tests for categorical variables.

Generalized Linear Mixed Models (GLMM) were used to analyze changes in HRQoL scores over time using the HRQoL scores as dependent variables and time (baseline, directly after treatment, and at follow-up 1 year later) as independent categorical variable. All models were adjusted for the following covariates: age, 
Fig. 1. SDS-BMI from baseline (T0) to directly after 1-year treatment (T1: 12 months after baseline) and 1-year follow-up after treatment (T2: 24 months after baseline). Error bars indicate standard error (SE) of the mean.

Hoedjes et al.: Health-Related Quality of Life in Children and Adolescents with Severe Obesity after Intensive Lifestyle Treatment and at 1-Year Follow-Up

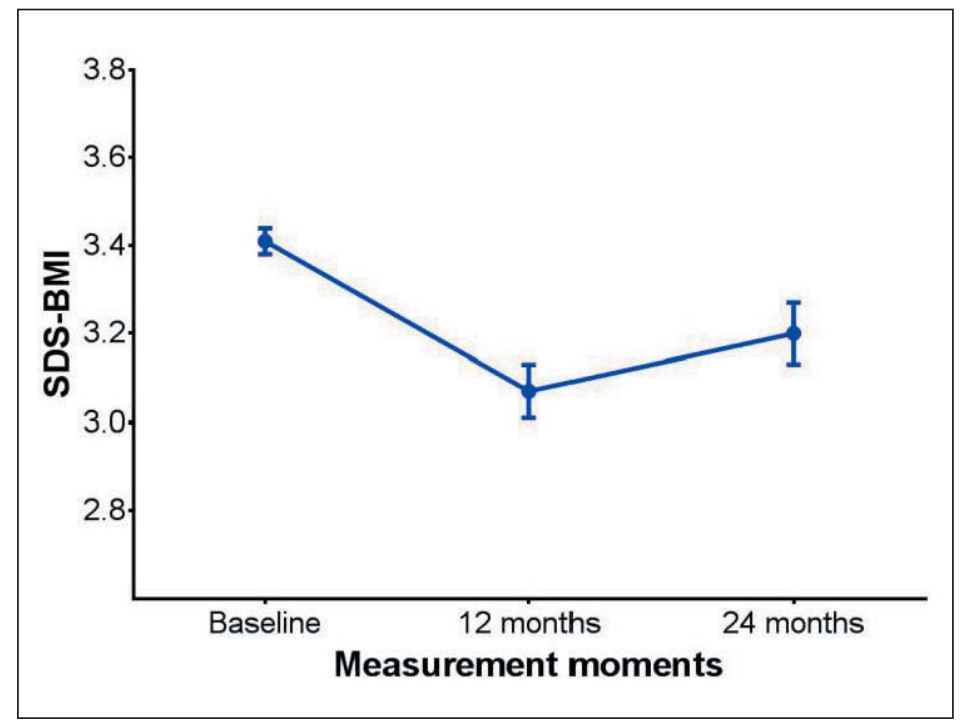

sex, ethnicity, educational level of the parents/caregivers, SES, household situation, and duration of inpatient treatment.

Partial correlations were used to examine associations between changes in SDS-BMI and changes in HRQoL. First, for SDS-BMI and for all overall HRQoL and domain scores, deltas were calculated from T0 to $\mathrm{T} 1$, from $\mathrm{T} 0$ to T2, and from T1 to T2. For each combination of deltas, a Pearson product-moment correlation coefficient was calculated. Pearson product-moment correlation coefficients were adjusted for the covariates mentioned above.

\section{Sensitivity Analyses}

Participants with complete data on the overall scores of the four HRQoL questionnaires (at T0, T1, and T2) were considered to have complete follow-up. If participants missed one or more overall scores of the four HRQoL questionnaires, participants were considered to have incomplete follow-up. In the sensitivity analysis, only participants with complete follow-up were included.

\section{Results}

Between August 2009 and July 2013, informed consent was obtained for 120 children and adolescents with severe obesity. Table 1 shows the baseline characteristics of the study population. Of the 120 participants, $99(82.5 \%)$ completed the intensive 1-year lifestyle treatment. Completers of treatment did not differ from dropouts of treatment $(\mathrm{N}=21)$ in any of the variables shown in table 1.

A significant reduction in mean SDS-BMI as compared to T0 was seen at $\mathrm{T} 1$, followed by partial regain of the lost weight at T2 (fig. 1).

\section{Changes in Generic and Weight-Related HRQoL}

Most generic and weight-related HRQoL scores increased statistically significantly from $\mathrm{T} 0$ to T1and from $\mathrm{T} 0$ to $\mathrm{T} 2$, whereas these scores did not change significantly from $\mathrm{T} 1$ to $\mathrm{T} 2$ (table 2).

The PedsQL 4.0 showed statistically significant improvements in all domains, with the largest improvements in the social functioning domain (beta $=11.9$ (95\% CI 7.83; 15.9) from T0 to T2). KIDSCREEN-52 scores particularly improved in the domains 'physical wellbeing' $($ beta $=4.16(95 \%$ CI 1.92; 6.39) ) and 'social acceptance and bullying' (beta $=6.17(95 \% \mathrm{CI}$ 


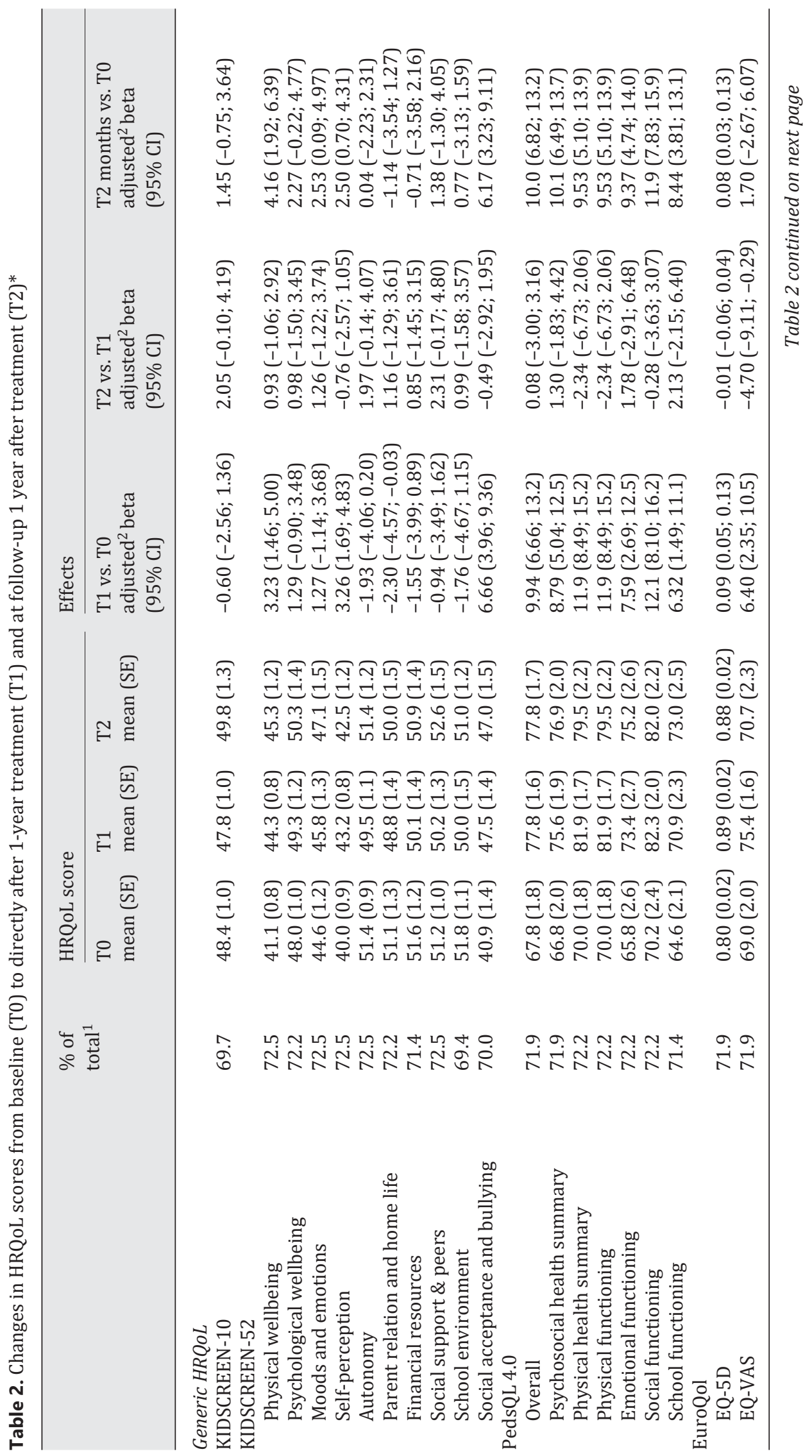


Hoedjes et al.: Health-Related Quality of Life in Children and Adolescents with Severe Obesity after Intensive Lifestyle Treatment and at 1-Year Follow-Up

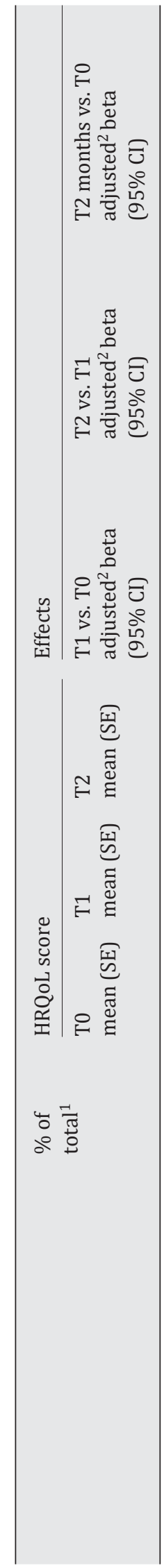

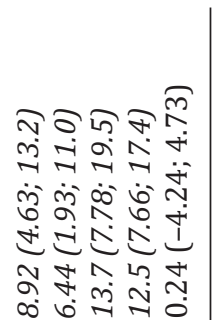

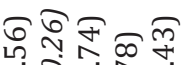

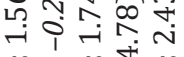

ने $\dot{\bar{n}} \ddot{\dot{m}} \dot{\mathrm{m}}$

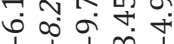

1 i 1 i

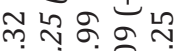

i

จุดจา

ㄱำ

ヘีヘ்ள்

mNㅗㄴ

ก 0

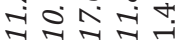

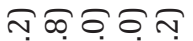

త్రతd

$1, \infty \infty x^{2}$

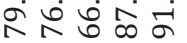

สำรส

ปతd త్రతd

궁

อค드

త্తd d d d

$\infty$ 十 $N$ เ $\infty$

윢ํㅇ

$\infty .6 \infty \sigma . \infty$

मीं

हี่

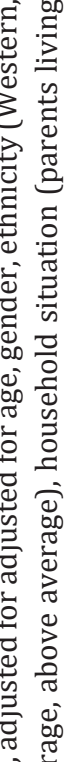

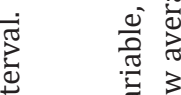

范

过

氙空

웡

ज

\%

:

ह

宊导

竞

추

ज्ञ

可导 ह

$\cong \cong$

웡

के 
Hoedjes et al:: Health-Related Quality of Life in Children and Adolescents with Severe Obesity after Intensive Lifestyle Treatment and at 1-Year Follow-Up

Table 3. Partial correlations between change in SDS-BMI and changes in HRQoL score over time ${ }^{\#}$

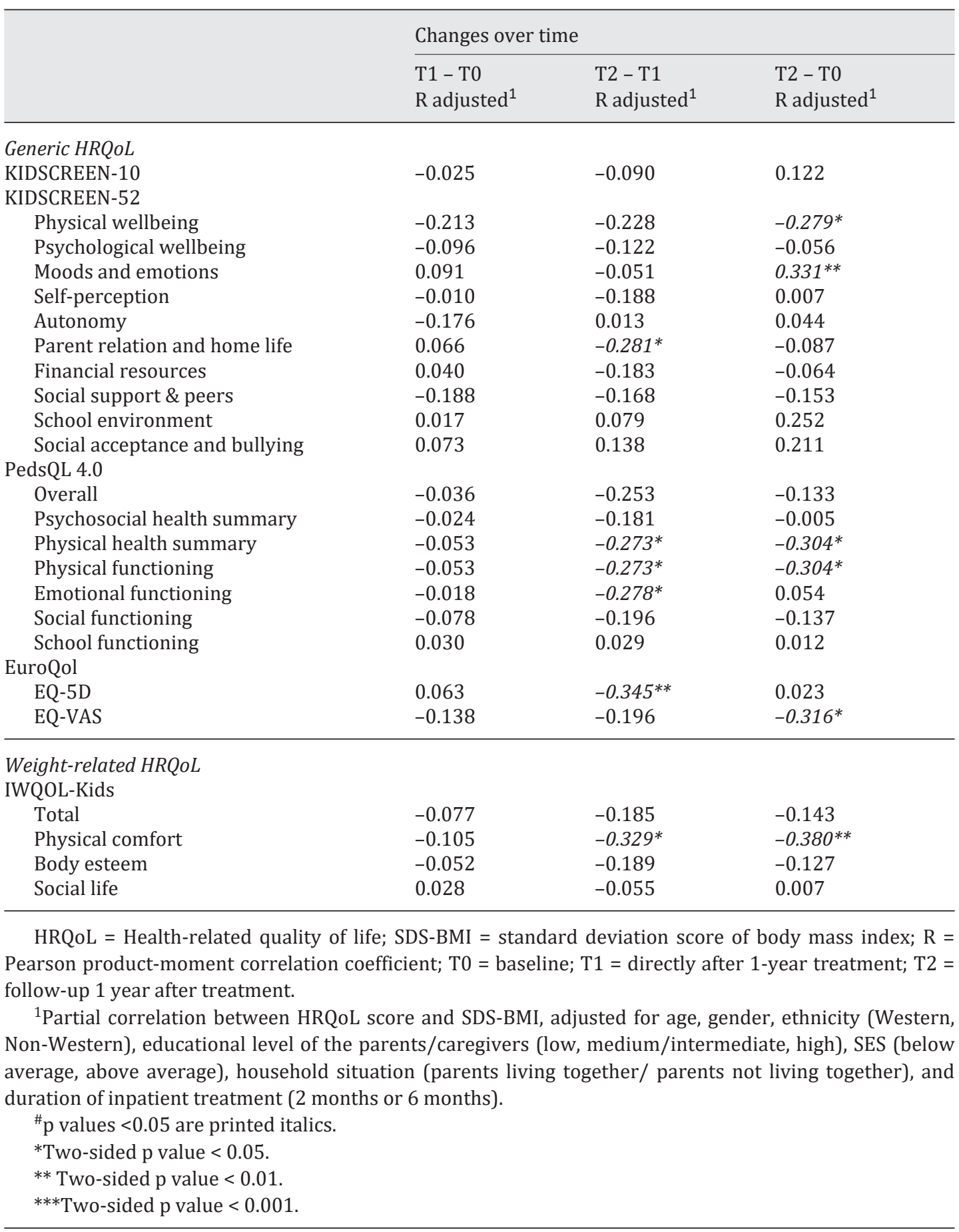

3.23; 9.11)). Although the EQ-5D-3L showed similar improvements, mean EQ-VAS score did not change from T0 to T2, but significantly increased from T0 to T1 (beta $=6.40$ (95\% CI 2.35; 10.5)), and significantly decreased from $\mathrm{T} 1$ to $\mathrm{T} 2$ (beta $=-4.70$ (95\% CI $-9.11 ;-0.29)$ ).

Weight-related HRQoL, as measured with the IWQOL-Kids, significantly improved in all domains, except for 'family relations'. The largest improvement was observed in the 'body esteem' domain (beta $=13.7$ (95\% CI: 7.78-19.5)). 


\section{Correlation between Changes in SDS-BMI and HRQoL}

Table 3 shows that larger weight loss from T0 to T2 was correlated with larger improvements in physical HRQoL domains of the KIDSCREEN-52 ( $<<0.05)$, the PedsQL $4.0(p<0.05)$, and the IWQOL-Kids $(\mathrm{p}<0.01)$. From T1 to T2, a larger increase in SDS-BMI was correlated with a larger decrease in the physical HRQoL domains of the PedsQL $4.0(p<0.05)$ and the IWQOL-Kids $(\mathrm{p}<0.05)$. Changes in generic and weight-related HRQoL from T0 to T1 were not correlated with changes in SDS-BMI.

\section{Sensitivity Analysis}

Analyses restricted to those who had complete follow-up ( $\mathrm{N}=53)$ showed slightly lower HRQoL scores at T0 and somewhat larger improvements, but conclusions remained essentially the same.

Similar results in the magnitude and directions of the correlations were found, although the level of significance sometimes changed because of the smaller statistical power.

\section{Discussion}

This study showed that children and adolescents with severe obesity experienced significant long-term improvements in generic and weight-related HRQoL after participating in an intensive 1-year lifestyle treatment, even when participants partially regained weight. Larger weight loss at 1-year follow-up after treatment was weakly to moderately correlated with larger improvements in physical HRQoL domains of all questionnaires.

To our knowledge, there are no other studies examining changes in HRQoL among children and adolescents with severe obesity participating in an intensive lifestyle treatment similar in the intensity of treatment and the length of follow-up. In addition, no other study assessed changes in all domains of generic and weight-related HRQoL. Furthermore, most other studies assessing the effects of lifestyle treatment in children and adolescents included either children and adolescents with obesity only or included both children and adolescents with obesity and with severe obesity, whereas only children and adolescents with severe obesity were included in our study [13, 14, 36-40].

The results of our study were generally in line with those found in other studies involving children and adolescents with less severe obesity, less intensive lifestyle treatment, and a shorter follow-up period [13, 14, 36-40]. In accordance with these other studies, we found that HRQoL scores generally increased during intensive lifestyle treatment and that these improvements were generally maintained at 1-year follow-up after treatment. Moreover, improvements at 1-year follow up after treatment in our study were clinically relevant for total weight-related HRQoL, weight-related 'body esteem' and 'social life' [41, 42], and for generic HRQoL overall, summary, and domain scores of the PedsQL 4.0, except for 'school functioning' [41, 43].

In accordance with our findings, several other studies showed statistically significant improvements in weight-related HRQoL directly after intensive lifestyle treatment $[15,37$, 38]. In line with our study, no improvements were observed on the 'family relations' domain $[37,38]$, suggesting that intensive lifestyle treatment does not influence weight-related HRQoL in the 'family relations' domain. However, our study did show a decrease in generic HRQoL on the 'parent relation and home life' domain during intensive lifestyle treatment as measured with the KIDSCREEN-52, suggesting that intensive lifestyle treatment does affect generic HRQoL in the 'parent relation and home life' domain.

Several other studies found similar improvements in generic HRQoL as assessed by the PedsQL 4.0 [37-39]. However, in contrast to our findings, they did not find improvements in 
the 'school functioning' domain. This discrepancy may be due to the use of shorter, less intensive lifestyle treatments compared with our study.

In our study, improvements in many psychosocial domains of the different HRQoL questionnaires were not related to the degree of weight loss, suggesting that weight loss is not necessary for improvements in these areas. This confirms findings from other studies [13, 14, 37]. Our finding that a reduction in SDS-BMI was correlated with an improvement in the physical HRQoL domain confirms findings from other studies examining the correlation between the degree of weight loss and improvement in generic HRQoL $[14,37,40]$. On the other hand, Rank et al. [13] did not find an association between changes in BMI and changes in the physical HRQoL domain. This lack of agreement between findings might be explained by the use of a different instrument to measure generic HRQoL (i.e. the German KINDL questionnaire).

This study has several strengths. It is unique with regard to the large number of participants, the intensity of the lifestyle treatment, and the long follow-up period after treatment. The 1-year intensive lifestyle treatment did not result in high dropout; 82.5\% (99 of the 120) of the participants completed treatment. The follow-up at 2 years after baseline provided the opportunity to observe that improvements in HRQoL were generally maintained, even after partial regain of the lost weight in the year after treatment. In addition, since multiple questionnaires were used to assess generic HRQoL, recommendations on the suitability of those questionnaires to measure changes in generic HRQoL in this particular population could be made.

This study also had some limitations. It was not possible to attribute the improvements in HRQoL to specific aspects of the intensive 1-year lifestyle treatment. To be able to determine whether intensive lifestyle treatment leads to improvements in HRQoL, as observed in this study, a randomized controlled trial should be conducted that includes a control group of children and adolescents with severe obesity receiving ambulatory obesity treatment. An additional limitation is the use of SDS-BMI as an outcome measure. SDS-BMI is a less reliable outcome measure to assess changes in body fatness in children and adolescents since it is influenced by pubertal stage [44]. SDS-BMI was chosen as an outcome measure because it is the most practically applicable. Finally, the EQ-5D-3L has not been validated for use in children and adolescents, and the IWQOL-kids has not been validated for use in children below the age of 11 . However, only a small proportion of our study sample was younger than 11 years (9\%). Furthermore, no difficulties in completing both questionnaires were encountered.

Our findings have some important implications for the practice. For healthcare professionals, healthcare insurance companies and policymakers, it is important to know that intensive lifestyle treatment for children and adolescents with severe obesity does not only lead to weight loss, but also to long-term improvements in generic and weight-related HRQoL, despite partial weight regain (e.g., with regard to decision making on reimbursement of the costs of intensive lifestyle treatment). For healthcare professionals involved in the treatment for children and adolescents with severe obesity, it is important to communicate these expected treatment effects to their patients. Our study confirms that generic and weightrelated HRQoL should both be included as outcome measures when evaluating lifestyle treatment in children and adolescents with severe obesity. The results of our study suggest that the IWQOL-kids is a suitable instrument to gain insight in changes in weight-related HRQoL in children and adolescents with severe obesity participating in a weight management program. In addition, our findings suggest that the PedsQL 4.0 is a sensitive instrument to measure changes in generic HRQoL in children and adolescents with severe obesity.

Further research is needed to assess potential differences in changes in generic and weight-related HRQoL between children and adolescents who do and those who do not regain weight after the end of intensive lifestyle treatment. This would provide valuable information for the treatment of children and adolescents with severe obesity. 
Hoedjes et al.: Health-Related Quality of Life in Children and Adolescents with Severe Obesity after Intensive Lifestyle Treatment and at 1-Year Follow-Up

\section{Conclusions}

This study is the first to report on long-term changes in HRQoL in children and adolescents with severe obesity participating in an intensive lifestyle treatment, and is unique with regard to the degree of obesity, the intensity of the lifestyle treatment, and the length of follow-up after treatment. It shows that children and adolescents with severe obesity experience significant long-term improvements in generic and weight-related HRQoL after participating in an intensive 1-year lifestyle treatment with an inpatient period. Although participants partially regained weight during the year following treatment, improvements in HRQoL were maintained. Larger long-term decreases in SDS-BMI were correlated with larger improvements in the physical HRQoL domains.

\section{Trial Registration}

Netherlands Trial Register (NTR): NTR1678, registered 20-Feb-2009.

\section{Acknowledgments}

This study was funded by the Netherlands Organization for Health Research and Development (ZonMw); 17099.2001. The funder did not have a role in the design and conduct of the study; the collection, management, analysis, and interpretation of the data; the preparation, review, or approval of the manuscript, and the decision to submit the manuscript for publication.

We are very grateful to our study participants and their parents/caregivers. We would also like to thank the professionals at Merem Childhood Obesity Center, Heideheuvel for their contribution to data collection. Finally, we would like to thank Ottelien van Weelden, MSc for contributing to the data collection and Michelle Belder, MSc for entering data in the database.

\section{Disclosure Statement}

Olga $\mathrm{H}$ van der Baan-Slootweg is affiliated with the treatment as pediatrician. Other than that the authors have no conflicts of interest to disclose.

\section{References}

1 Franks PW, Hanson RL, Knowler WC, Sievers ML, Bennett PH, Looker HC: Childhood obesity, other cardiovascular risk factors, and premature death. N Engl J Med 2010;362:485-493.

2 Ludwig DS: Childhood obesity - the shape of things to come. N Engl J Med 2007;357:2325-2327.

3 Makkes S, Renders CM, Bosmans JE, van der Baan-Slootweg OH, Seidell JC: Cardiometabolic risk factors and quality of life in severely obese children and adolescents in the Netherlands. BMC Pediatr 2013;13:62.

4 Buttitta M, Iliescu C, Rousseau A, Guerrien A: Quality of life in overweight and obese children and adolescents: a literature review. Qual Life Res 2014;23:1117-1139.

5 Puder JJ, Munsch S: Psychological correlates of childhood obesity. Int J Obes (Lond) 2010;34(suppl 2):S37-43.

6 van Wijnen LG, Boluijt PR, Hoeven-Mulder HB, Bemelmans WJ, Wendel-Vos GC: Weight status, psychological health, suicidal thoughts, and suicide attempts in Dutch adolescents: results from the 2003 E-MOVO project. Obesity (Silver Spring) 2010;18:1059-1061.

-7 Kelly AS, Barlow SE, Rao G, Inge TH, Hayman LL, Steinberger J, Urbina EM, Ewing LJ, Daniels SR; on behalf of the American Heart Association Atherosclerosis, Hypertension, and Obesity in the Young Committee of the Council on Cardiovascular Disease in the Young, Council on Nutrition, Physical Activity and Metabolism, and Council on Clinical Cardiology: Severe obesity in children and adolescents: identification, associated health risks, and treatment approaches: a scientific statement from the American Heart Association. Circulation 2013;128:1689-1712.

8 Schwimmer JB, Burwinkle TM, Varni JW: Health-related quality of life of severely obese children and adolescents. JAMA 2003;289:1813-1819. 
Hoedjes et al.: Health-Related Quality of Life in Children and Adolescents with Severe Obesity after Intensive Lifestyle Treatment and at 1-Year Follow-Up

-9 Testa MA, Simonson DC: Assessment of quality of life outcomes. N Engl J Med 1996;334:833-840.

10 Tsiros MD, Olds T, Buckley JD, Grimshaw P, Brennan L, Walkley J, Hills AP, Howe PR, Coates AM: Health-related quality of life in obese children and adolescents. Int J Obes (Lond) 2009;33:387-400.

-11 Griffiths LJ, Parsons TJ, Hill AJ: Self-esteem and quality of life in obese children and adolescents: a systematic review. Int J Pediatr Obes 2010;5:282-304.

12 Kelly KP, Kirschenbaum DS: Immersion treatment of childhood and adolescent obesity: the first review of a promising intervention. Obes Rev 2011;12:37-49.

13 Rank M, Wilks DC, Foley L, Jiang Y, Langhof H, Siegrist M, Halle M: Health-related quality of life and physical activity in children and adolescents 2 years after an inpatient weight-loss program. J Pediatr 2014;165: 732-737 e2.

14 Fullerton G, Tyler C, Johnston CA, Vincent JP, Harris GE, Foreyt JP: Quality of life in Mexican-American children following a weight management program. Obesity (Silver Spring) 2007;15:2553-2556.

15 Kolotkin RL, Zeller M, Modi AC, Samsa GP, Quinlan NP, Yanovski JA, Bell SK, Maahs DM, de Serna DG, Roehrig HR: Assessing weight-related quality of life in adolescents. Obesity (Silver Spring) 2006;14:448-457.

16 Vos RC, Huisman SD, Houdijk EC, Pijl H, Wit JM: The effect of family-based multidisciplinary cognitive behavioral treatment on health-related quality of life in childhood obesity. Qual Life Res 2012;21:1587-1594.

17 Johnston CA, Tyler C, Palcic JL, Stansberry SA, Gallagher MR, Foreyt JP: Smaller weight changes in standardized body mass index in response to treatment as weight classification increases. J Pediatr 2011;158:624-627.

18 Danielsson P, Kowalski J, Ekblom Ö, Marcus C: Response of severely obese children and adolescents to behavioral treatment. Acrch Pediatr Adolesc Med 2012;166;1103-1108.

19 Patrick DL, Skalicky AM, Edwards TC, Kuniyuki A, Morales LS, Leng M, Kirschenbaum DS: Weight loss and changes in generic and weight-specific quality of life in obese adolescents. Qual Life Res 2011;20:961-968.

20 Halberstadt J, Makkes S, de Vet E, Jansen A, Nederkoorn C, van der Baan-Slootweg OH, Seidell JC: The role of self-regulating abilities in long-term weight loss in severely obese children and adolescents undergoing intensive combined lifestyle interventions (HELIOS); rationale, design and methods. BMC Pediatr 2013;13:41.

-21 Makkes S, Halberstadt J, Renders CM, Bosmans JE, van der Baan-Slootweg OH, Seidell JC: Cost-effectiveness of intensive inpatient treatments for severely obese children and adolescents in the Netherlands; a randomized controlled trial (HELIOS). BMC Public Health 2011;11:518.

22 Ravens-Sieberer U, Gosch A, Rajmil L, Erhart M, Bruil J, Power M, et al: The KIDSCREEN-52 quality of life measure for children and adolescents: psychometric results from a cross-cultural survey in 13 European countries. Value Health 2008;11:645-658.

23 The KIDSCREEN Group Europe: The KIDSCREEN Questionnaires. Quality of Life Questionnaires for Children and Adolescents. Handbook. Lengerich, Pabst Science Publishers, 2006.

-24 Ravens-Sieberer U, Erhart M, Rajmil L, Herdman M, Auquier P, Bruil J, Pwer M, Duer W, Abel T, Czemy L, Mazur J, Czimbalmos A, Tountas Y, HagquistC, KilroeJ: Reliability, constructand criterion validity of the KIDSCREEN-10 score: a short measure for children and adolescents' well-being and health-related quality of life. Qual Life Res 2010;19:1487-1500.

25 Varni JW, Seid M, Kurtin PS: PedsQL 4.0:reliability and validity of the Pediatric Quality of Life Inventory version 4.0 generic core scales in healthy and patient populations. Med Care 2001;39:800-812.

26 EuroQol - a new facility for the measurement of health-related quality of life. Health Policy 1990;16:199-208.

27 Lamers LM, Stalmeier PF, McDonnell J, Krabbe PF, van Busschbach JJ: Measuring the quality of life in economic evaluations: the Dutch EQ-5D tariff (in Dutch). Ned Tijdschr Geneeskd 2005;149:1574-1578.

28 Wouters EJM: Suffering from obesity. Psychosocial aspects of assessment, treatment, and aetiology. PhD Thesis, Tilburg University, 2010.

29 Engelen V, Haentjens MM, Detmar SB, Koopman HM, Grootenhuis MA: Health related quality of life of Dutch children: psychometric properties of the PedsQL in the Netherlands. BMC Pediatr 2009;9:68.

-30 Wouters EJM, Geenen R, Kolotkin RL, Vingerhoets AJJM: Met lichaamsgewicht samenhangende kwaliteit van leven bij adolescenten. Psychometrische kwaliteit van de Nederlandse vertaling van de IWQOL-Kids. Tijdschr Kindergeneeskund 2010;3:119-125.

31 CBS: Statistisch jaarboek 2000. Heerlen, Statistics Netherlands (CBS), 2000.

-32 de Jong E, Schokker DF, Visscher TL, Seidell JC, Renders CM: Behavioural and socio-demographic characteristics of Dutch neighbourhoods with high prevalence of childhood obesity. Int J Pediatr Obes 2011;6:298-305.

33 Kardal M, Lodder B: De gezonde levensverwachting naar sociaaleconomische status. Den Haag, Centraal Bureau voor de Statistiek, 2008.

34 Knol FA: De sociaal-ruimtelijke ontwikkeling van wijken tussen 1971 en 1995. Den Haag, Sociaal Cultureel Planbureau,1998.

35 Growth Analyser (computer program). Stichting Kind en Groei, 2010.

-36 Hofsteenge GH, Weijs PJ, Delemarre-van de Waal HA, de Wit M, Chinapaw MJ: Effect of the Go4it multidisciplinary group treatment for obese adolescents on health related quality of life: a randomised controlled trial. BMC Public Health 2013;13:939.

-37 Quinlan NP, Kolotkin RL, Fuemmeler BF, Costanzo PR: Psychosocial outcomes in a weight loss camp for overweight youth. Int J Pediatr Obes 2009;4:134-142.

38 Wong WW, Barlow SE, Mikhail C, Wilson TA, Hernandez PM, Shypailo RJ, Abrams SH: A residential summer camp can reduce body fat and improve health-related quality of life in obese children. J Pediatr Gastroenterol Nutr 2013;56:83-85. 
Hoedjes et al.: Health-Related Quality of Life in Children and Adolescents with Severe Obesity after Intensive Lifestyle Treatment and at 1-Year Follow-Up

-39 Yackobovitch-Gavan M, Nagelberg N, Phillip M, Ashkenazi-Hoffnung L, Hershkovitz E, Shalitin S: The influence of diet and/or exercise and parental compliance on health-related quality of life in obese children. Nutr Res 2009;29:397-404.

40 Poeta LS, Duarte Mde F, Giuliano Ide C, Mota J: Interdisciplinary intervention in obese children and impact on health and quality of life. J Pediatr (Rio J) 2013;89:499-504.

-41 Jaeschke R, Singer J, Guyatt GH: Measurement of health status. Ascertaining the minimal clinically important difference. Control Clin Trials 1989;10:407-415.

42 Modi AC, Zeller MH: The IWQOL-Kids ${ }^{\circledR}$ : establishing minimal clinically important difference scores and testretest reliability. Int J Pediatr Obes 2011;6:e94-e96.

43 Varni JW, Burwinkle TM, Seid M, Skarr D: The PedsQL 4.0 as a pediatric population health measure: feasibility, reliability, and validity. Ambul Pediatr 2003;3:329-341.

44 Demerath EW, Schubert CM, Maynard LM, Sun SS, Chumlea WC, Pickoff A, Czerwinski SA, Towne B, Siervogel RM: Do changes in body mass index percentile reflect changes in body composition in children? Data from the Fels Longitudinal Study. Pediatrics 2006;117:e487-495. 